\title{
Energy Transfer from Perovskite Nanocrystals to Dye Molecules Does Not Occur by FRET
}

\author{
Felix J. Hofmann, ${ }^{\dagger}$ Maryna I. Bodnarchuk, ${ }^{\ddagger, \S}$ Dmitry N. Dirin, ${ }^{\ddagger}, \S \odot$ Jan Vogelsang, ${ }^{\dagger}$

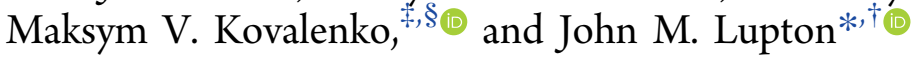 \\ ${ }^{\dagger}$ Institut für Experimentelle und Angewandte Physik, Universität Regensburg, Universitätsstraße 31, 93053 Regensburg, Germany \\ ${ }^{\ddagger}$ ETH Zürich, Department of Chemistry and Applied Biosciences, Vladimir Prelog Weg 1, CH-8093 Zürich, Switzerland \\ ${ }^{\S}$ Empa - Swiss Federal Laboratories for Materials Science and Technology, Überlandstr. 129, CH-8600 Dübendorf, Switzerland
}

ABSTRACT: Single formamidinium lead bromide $\left(\mathrm{FAPbBr}_{3}\right)$ perovskite nanocubes, approximately $10 \mathrm{~nm}$ in size, have extinction cross sections orders of magnitude larger than single dye molecules and can therefore be used to photoexcite one single dye molecule within their immediate vicinity by means of excitation-energy transfer (EET). The rate of photon emission by the single dye molecule is increased by 2 orders of magnitude under excitation by EET compared to direct excitation at the same laser fluence. Because the dye cannot accommodate biexcitons, NC biexcitons are filtered out by EET, giving rise to up to

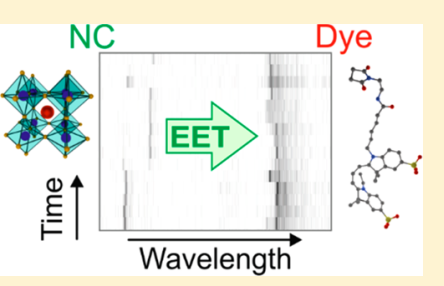
an order-of-magnitude improvement in the fidelity of photon antibunching. We demonstrate here that, contrary to expectation, energy transfer from the nanocrystal to dye molecules does not depend on the spectral line widths of the donor and acceptor and is therefore not governed by Förster's theory of resonance energy transfer (FRET). Two different cyanine dye acceptors with substantially different spectral overlaps with the nanocrystal donor show a similar lightharvesting capability. Cooling the sample from room temperature to $5 \mathrm{~K}$ reduces the average transition line widths 25 -fold but has no apparent effect on the number of molecules emitting, i.e., on the spatial density of single dye molecules being photoexcited by single nanocrystals. Narrow zero-phonon lines are identified for both donor and acceptor, with an energetic separation of over 40 times the line width, implying a complete absence of spectral overlap-even though EET is evident. Both donor and acceptor exhibit spectral fluctuations, but no correlation is apparent between the jitter, which controls spectral overlap, and the overall light harvesting. We conclude that the energy transfer process is fundamentally nonresonant, implying effective energy dissipation in the perovskite donor because of strong electron-phonon coupling of the carriers comprising the exciton. The work highlights the importance of performing cryogenic spectroscopy to reveal the underlying mechanisms of energy transfer in complex donor-acceptor systems.

KEYWORDS: Perovskites, $\mathrm{FAPbBr}_{3}$ nanocrystals, single-molecule spectroscopy, nonresonant energy transfer

$\mathrm{T}$ here are many model systems of natural light-harvesting phenomena, which exploit resonant dipole-dipole coupling to enable excitation-energy transfer (EET). ${ }^{1,2}$ To a first approximation, in the framework of point dipoles, the rate of EET is given by Förster's theory of resonance energy transfer (FRET) and depends on the relative orientation and spatial separation of transition dipoles and the spectral overlap between the fluorescence of the donor and the absorption of the acceptor. In molecules, this spectral overlap arises both from the homogeneous broadening of the electronic transitions as well as from the coupling to vibrations. ${ }^{1,3}$ Depending on the strength of vibrational coupling, as in many cases of molecular donor-acceptor complexes, EET is not strictly resonant in terms of the electronic transitions, but involves additional molecular vibrations. These vibrations broaden both the donor emission and the acceptor absorption spectrum, increasing spectral overlap. However, nonresonant dipole-dipole coupling may also occur without direct electron-phonon coupling in the optical transitions of donor and acceptor. ${ }^{4-8}$ This situation is pronounced in rare-earth-doped crystals, where luminescence and absorption are characterized by narrow atomic-like transitions, yet dipole-dipole coupling and down- hill EET arise because the crystal host can absorb the energy difference in the form of a phonon. ${ }^{9-11}$ In such polar crystals, Fröhlich phonons arise, which exhibit features of an anharmonic interatomic binding potential and often also support multiple-phonon transitions. ${ }^{12,13}$

A particularly interesting situation arises when inorganic nanostructures are dipole-coupled to organic dye molecules. $^{14-22}$ Semiconductor nanoparticles have molar extinction cross sections orders of magnitude larger than those of common dye molecules and can therefore absorb light and transfer excitation energy to a dye molecule in close proximity. ${ }^{23}$ The nanoparticle then acts as an excitonic antenna for the dye. This effect has been well studied in CdSe nanoparticles ${ }^{23,24}$ but also arises in the combination of perovskite nanoparticles and dye molecules. ${ }^{25}$ The degree of spectral overlap between CdSe nanocrystal (NC) donor emission and dye acceptor absorption can be tuned by

Received: September 12, 2019

Revised: October 9, 2019

Published: October 24, 2019 
changing the temperature. ${ }^{26,27}$ Alternatively, the Stark shift induced by an external electric field can move the NC-dye pair in and out of resonance. ${ }^{23}$ In both cases, EET appears to arise due to FRET, with a clear reduction in EET rates as the degree of resonance is reduced.

Here, we examine the effect of donor and acceptor transition line widths in $\mathrm{FAPbBr}_{3} \mathrm{NC}$-dye pairs. In contrast to conventional II-VI semiconductor nanocrystals, EET appears to be virtually independent of the degree of spectral overlap, implying the occurrence of highly efficient nonresonant EET. Since Raman scattering reveals effective multiphonon transitions in perovskite $\mathrm{NCs},{ }^{12,13}$ we propose that effective multiphonon emission can occur in the NC prior to EET, enabling dipole-dipole coupling even in the absence of appreciable spectral overlap between donor and acceptor. We recently demonstrated efficient light harvesting from single perovskite NCs to single dye molecules, allowing us to pick out molecules from an ensemble with a mean intermolecular spacing 2 orders of magnitude below that of typical singlemolecule concentrations. ${ }^{25}$ Light harvesting is so efficient that it even occurs to the triplet excited state of the dye, inducing reverse intersystem crossing from a higher-lying doubly excited triplet state. ${ }^{25}$ Since dye molecules, in contrast to NCs, can only support one single excited state at a time, NC biexcitons ${ }^{28}$ are efficiently filtered out in the light-harvesting process, giving rise to substantially improved photon antibunching. ${ }^{25}$ Given the similarity of those processes to light harvesting involving CdSe-based NCs, ${ }^{24}$ it is tempting to attribute EET to FRET. But is this assignment really supported by a close inspection of the degree of resonance between donor and acceptor, the transition line widths, and the spectral overlaps?

We previously studied EET from single $\mathrm{FAPbBr}_{3} \mathrm{NCs}, 10 \times$ $10 \times 10 \mathrm{~nm}^{3}$ in size, to single molecules of the cyanine dye Cy3. ${ }^{25}$ Following earlier assignments in the literature, ${ }^{21}$ we attributed this EET to FRET without further analysis. However, to conclusively establish FRET as the EET mechanism it is crucial to vary either the donor-acceptor spacing, which is often very challenging to do, or to change the spectral overlap integral of donor emission and acceptor absorption. At room temperature, in the ensemble, the NC donor emission and the dye acceptor absorption display appreciable overlap, corresponding to an overlap integral of 5.8 $\times 10^{12} \mathrm{~nm}^{4} \mathrm{M}^{-1} \mathrm{~cm}^{-1}$. Figure 1 shows results of the same experiment reported in ref 25, but using the longer-wavelength dye Cy5 instead. Panel (a) plots the absorption and photoluminescence (PL) spectra of NCs and the dye in toluene solution, at room temperature, with a representative high-resolution transmission electron microscope image of a single crystal inset in panel (b). For this combination of materials, the overlap integral has a value of $4.3 \times 10^{11} \mathrm{~nm}^{4}$ $\mathrm{M}^{-1} \mathrm{~cm}^{-1}$, over an order of magnitude lower than that for the $\mathrm{NC} / \mathrm{Cy} 3$ combination. The spectra in the ensemble are inhomogeneously broadened, so when considering single particles and molecules, the microscopic effective spectral overlap between one single donor and one single acceptor varies strongly. ${ }^{3,27,29}$ It is very challenging to measure the dye absorption spectrum directly on the single-molecule level, but, based on the Franck-Condon principle, the single-molecule PL spectrum of the dye can provide a reasonable estimate. Panel (b) shows five different PL spectra of pairs of NCs and single Cy5 dye molecules immobilized in a spin-coated poly(methyl methacrylate) (PMMA) matrix, normalized in intensity to the NC spectrum. Some scatter of the single NC
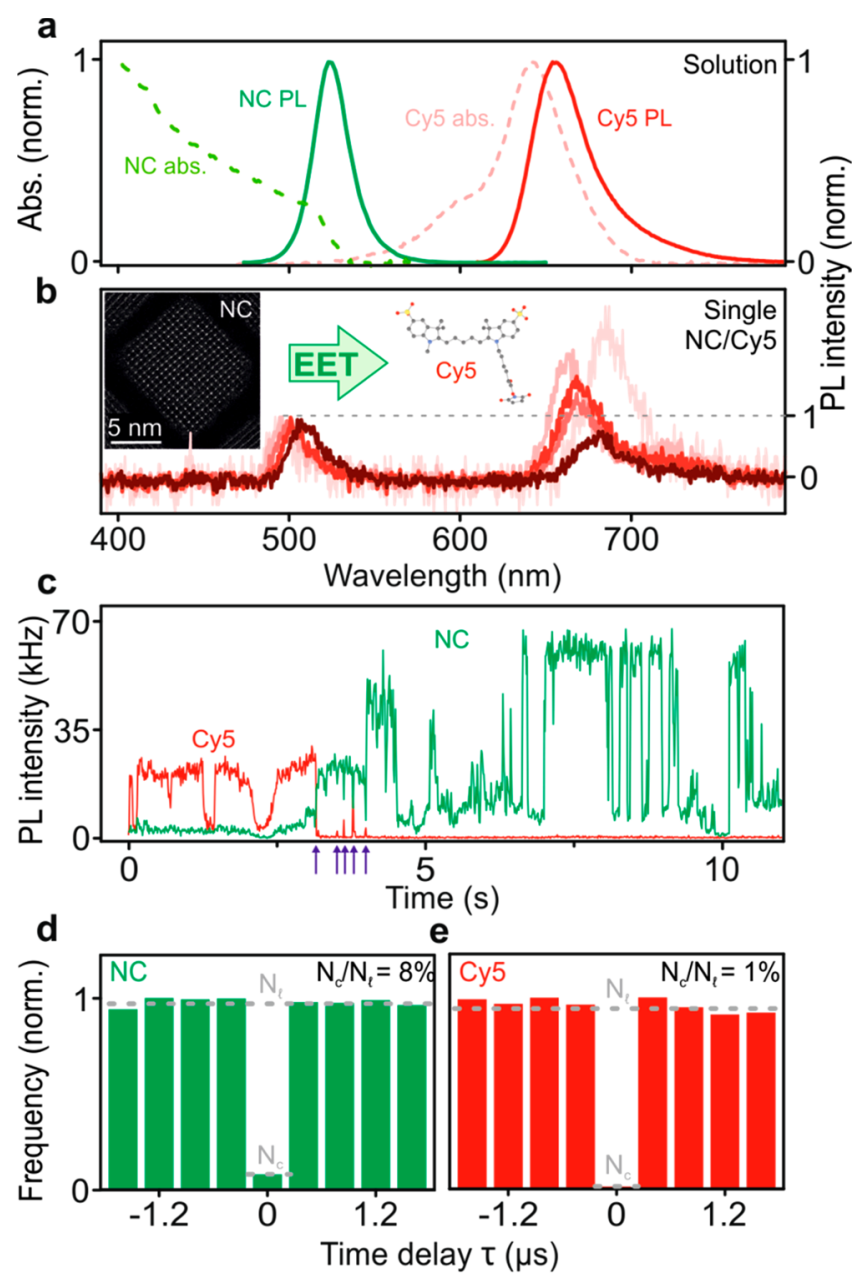

Figure 1. Excitation-energy transfer (EET) from single $\mathrm{FAPbBr}_{3} \mathrm{NCs}$ to single $\mathrm{Cy} 5$ dye molecules at room temperature. (a) Ensemble absorption and PL spectra of NCs and Cy5 in toluene solution. There is only minimal overlap between dye absorption and NC emission spectra. (b) PL spectra of single NC-Cy5 pairs showing EET, immobilized in a PMMA film, under excitation in the NC absorption, normalized to the NC emission. The inset shows a HRTEM image of a single NC. (c) PL intensity transient of a single NC/Cy5 pair under excitation of the NC. The PL is split between NC (green) and Cy5 (red) channels by a $600 \mathrm{~nm}$ dichroic mirror. After bleaching of the Cy5 acceptor at time $4 \mathrm{~s}$, EET is blocked and the NC emission increases. The arrows mark periods of anticorrelation between the two intensity channels. (d) Second-order intensity correlation function, $g^{(2)}(\tau)$, of the emission of a single NC shown in the intensity trace in panel (c). The photon coincidence ratio of central to lateral peaks in the histogram, $N_{\mathrm{c}} / N_{\mathrm{b}}$, shows a photon antibunching dip down to $8 \%$. This finite coincidence ratio arises from NC biexciton emission. (e) Photon antibunching from the single Cy5 molecule of the donoracceptor pair discussed in panel (c), excited by EET. The NC biexciton emission is removed by EET to the molecule, lowering $g^{(2)}(0)$ to $1 \%$, which implies perfect deterministic single-photon emission.

spectra is observed around $500 \mathrm{~nm},{ }^{30}$ but the variation of single-molecule spectra is much larger, ranging from 665 to $690 \mathrm{~nm}$. A laser at $405 \mathrm{~nm}$ is used for photoexcitation. At this wavelength, the $\mathrm{Cy} 5$ dye cannot be excited directly, and PL from the dye occurs because of EET from the NC to the dye. EET is not perfect since both dye emission and residual NC emission are observed simultaneously. On the level of single particles and molecules, there is no discernible overlap 
between the two PL spectra, yet there also appears to be no obvious correlation of the relative dye acceptor intensity with the spectral position of the acceptor PL relative to the donor PL spectrum. Such a lack of correlation between spectral features, even in terms of the PL rather than the absorption, is very unusual for single donor-acceptor complexes. ${ }^{31}$

The fact that donor and acceptor are interlinked is apparent from the photon dynamics of the two PL channels, resolved in green for a $\mathrm{NC}$ and in red for a $\mathrm{Cy} 5$ molecule in Figure 1c. There are periods of clear anticorrelation in the two channels as marked by blue arrows. As the dye emission is quenched, the NC emission resumes, and vice versa. After $4 \mathrm{~s}$, the dye emission appears dormant, presumably because the molecule has photobleached. Blinking of the NC emission then has no effect on the dye.

The most dramatic signature of the consequence of EET from the NC to the dye comes from consideration of the photon statistics, which are measured by passing the PL through a beam splitter on each spectral detection channel and measuring the intensity autocorrelation as a function of the delay time $\tau$ between two detectors on either side of the beam splitter. Since a pulsed laser is used for excitation with a repetition period of $400 \mathrm{~ns}$, photon coincidences appear as bars in the histogram in Figure 1d, e. The photon antibunching ratio, $N_{c} / N_{1}$, is defined as the ratio between central, $N_{c}$, and lateral peaks, $N_{1}$, of the histograms. Since biexcitons can form in the single $\mathrm{NC}{ }^{32}$ sequential emission of two photons can arise within the time resolution of the experiment, raising the central coincidence peak to $8 \%$ (panel d). This residual photon correlation virtually vanishes when only the photon statistics of the dye emission are considered in panel (e). The antibunching ratio now drops to $1 \%$, which, given the finite signal-to-noise ratio of the experiment, constitutes near-perfect photon antibunching. Because the dye molecule cannot accommodate biexcitons, these species are effectively gated out in the EET process. ${ }^{25}$

It is surprising that FRET from $\mathrm{FAPbBr}_{3} \mathrm{NCs}$ to single dye molecules works equally well for $\mathrm{Cy} 3$ and $\mathrm{Cy} 5$ acceptor dyes, even though the spectral overlap between donor emission and acceptor absorption is very different for these two acceptors. An easier way to tune the spectral overlap, instead of exploring different acceptor materials, is to vary the sample temperature, which directly affects donor and acceptor PL line widths. ${ }^{3,23,29}$ Since the ensemble spectra are inhomogeneously broadened, a reduction of temperature has only a modest effect, provided that the energetic disorder of donor and acceptor does not change with temperature. To assess the effect of temperature on the intrinsic transition line width, we consider many different single-molecule and single-particle spectra and average these by shifting them to the mean peak wavelength. Since the spectrometer sensitivity is reduced at wavelengths above $650 \mathrm{~nm}$, we perform the following experiments with a Cy3 acceptor rather than with Cy5. Figure 2a shows the results of this procedure for 20 single pairs of $\mathrm{NC}$ and dye in which both show emission. On average, at $200 \mathrm{~K}$, there is little overlap between the NC and dye spectra. The effect becomes much more dramatic at $5 \mathrm{~K}$, where the mean zero-phonon line of the NC narrows to 0.7 at $538 \mathrm{~nm}\left(24.1 \mathrm{~cm}^{-1}\right.$ spectral width); that of the Cy3 dye narrows to 0.2 at $575 \mathrm{~nm}$ (corresponding to a width of $6.0 \mathrm{~cm}^{-1}$ ).

To examine what effect, if any, transition line widths have on EET, we consider the spatial density of single dye molecules excited by EET. The insets in panels (a) and (b) show the a
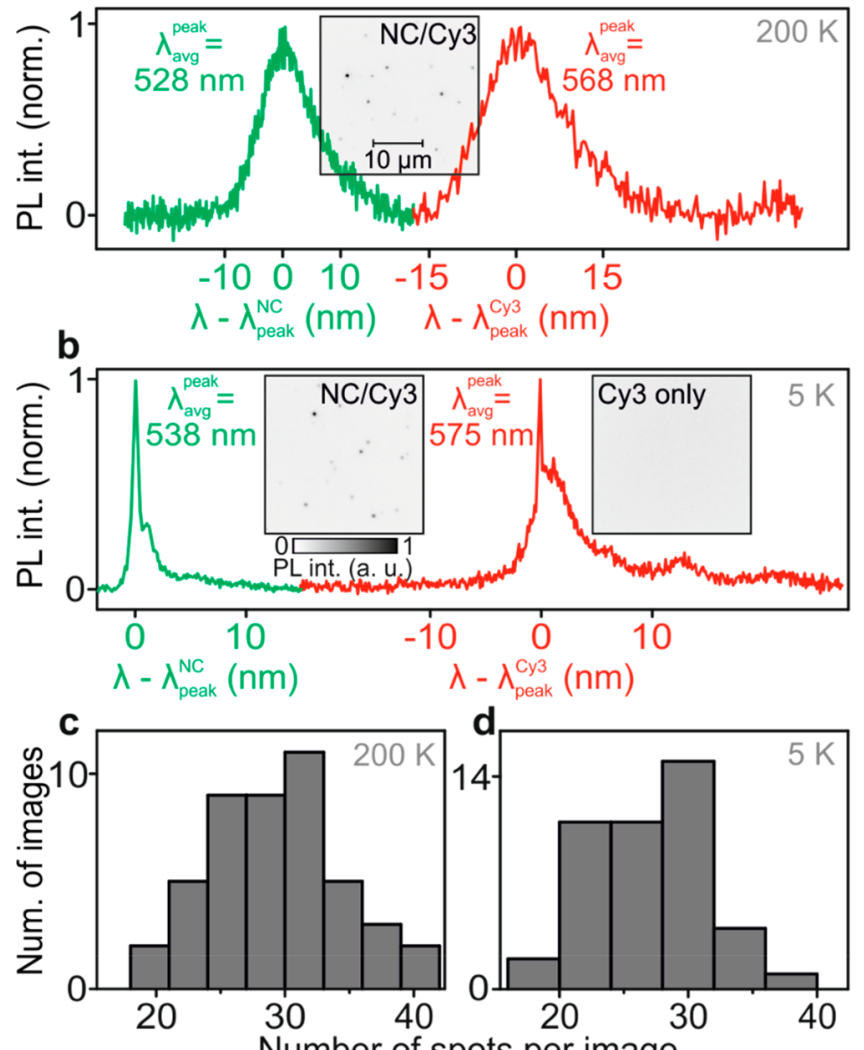

Number of spots per image

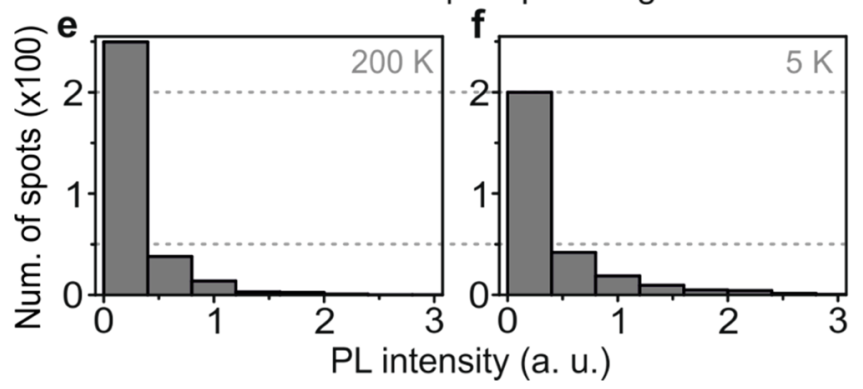

Figure 2. Temperature dependence of donor-acceptor PL spectra and of EET from single NCs to single Cy3 dye molecules. (a, b) Temperature-dependent average single-particle (green) and singlemolecule (red) PL spectra. To remove the effect of inhomogeneous broadening in the averaging of single-molecule and single-particle spectra, each single spectrum was shifted from its individual peak position $\lambda_{\text {peak }}$ to the average peak position $\lambda_{\text {avg }}^{\text {peak }}$ stated in the figure. The dye spectra narrow by a factor of 25 upon cooling and the NC spectra narrow 15 -fold, implying a dramatic reduction in spectral overlap between donor and acceptor. Despite this narrowing, the density of spots in the fluorescence microscope images (inset) due to single dye molecules excited by EET does not change. To acquire these images, a $550 \mathrm{~nm}$ long-pass filter was used, cutting out the NC PL. No single-molecule emission is observed in the absence of NC absorbers [inset in the right of panel (b)], implying that the dye cannot be excited directly under these conditions. The microscope images in (a) and (b) were recorded under the same camera settings, and the intensity scalebar is shown beneath. (c) Histogram of the number of single-molecule spots per image for $50 \mathrm{PL}$ images each at 200 and $5 \mathrm{~K}$ (d). (e, f) Histograms of intensities of PL spots in (c) and (d), which are also similar for both temperatures.

density of single-molecule fluorescence spots, measured with a long-pass filter in the microscope, which only passes the Cy3 emission and not the NC PL. Surprisingly, even though the 
effective spectral line widths of donor and acceptor narrow by a factor of approximately 25 upon cooling from 200 to $5 \mathrm{~K}$, there is no discernible difference in the density of $\mathrm{Cy} 3$ molecules observed in the microscope. Panels (c) and (d) show the statistics of spot density for 50 single images each, which are unaffected by temperature. Likewise, the distribution of intensities of single-molecule emission is not dependent on temperature, as shown in panels (e) and (f). It is crucial to confirm that all dye molecules really are excited by EET and are not excited directly by the laser. The right image inset in panel (b) shows an analogous dye-only sample, excited under the same conditions. In the absence of NCs, no PL from single dye molecules is observed.

It is not immediately obvious whether EET always occurs to one and the same acceptor molecule or whether different dye molecules in the vicinity of the donor act as the acceptor. If several dye molecules act as acceptors, then weak fluctuations in donor-acceptor coupling strength could conceivably be compensated by activating different EET pathways. We can exclude this possibility by considering the arrangement of the donor-acceptor system, which is accessible by measuring the polarization anisotropy in fluorescence, shown in Figure 3. The $\mathrm{PL}$ is passed through a half-wave plate and split into its two polarization components by a Wollaston prism. As the halfwave plate is rotated, the ratio of the two polarization components changes, provided that the PL was originally polarized. Panel (b) shows the result for a single NC-dye pair at $5 \mathrm{~K}$. Evidently, the $\mathrm{PL}$ of the $\mathrm{NC}$ is unpolarized, whereas the $\mathrm{PL}$ of the single molecule is linearly polarized. By fitting a version of Malus' law, stated in panel (a), to the modulation of the PL intensity, a modulation depth $M$ is extracted for each pair, with 0 corresponding to unpolarized emission and 1 to linearly polarized emission. The histogram in panel (c) for 300 donor-acceptor pairs shows that, on average, the single NC is unpolarized in emission whereas the single dye molecule emits polarized light. If the NC did transfer energy to different single dye molecules in its vicinity, different effective dipole orientations would arise so that, on average, the dye emission would not be polarized. Such a depolarization evidently does not arise here; EET turns unpolarized NC emission into polarized dye emission. Obviously, each single dye molecule has a different orientation with regards to the setup. The phase angle of the sinusoidal modulation in panel (b) is therefore different for every single dye molecule and scatters uniformly between $0^{\circ}$ and $90^{\circ}$ as shown in the inset of panel (c).

Finally, we consider the role of spectral dynamics of the single donor and acceptor, i.e., the spectral diffusion of the electronic transitions. Figure 4 indicates the level of spectral scatter of single NCs and single dye molecules, selected from over 300 single spectra at $5 \mathrm{~K}$. In this case, the spectra do not arise from the same NC-dye pair. Narrow transitions of the NC are seen to scatter between 534 and $546 \mathrm{~nm}$, and the zerophonon lines of the dye molecules span a similar range from 562 to $578 \mathrm{~nm}$. Both spectra show some substructure. The second peak in the NC spectra is presumably associated with the formation of a biexciton or trion, ${ }^{32}$ whereas the various lower-energy transitions in the dye molecules can be attributed to the different vibrational modes. Single-photon emitters at low temperature such as NCs or dye molecules often feature subtle spectral dynamics, which may resemble a continuous diffusion or discrete jumps. ${ }^{33}$ Three examples of such dynamics are shown in panels $(b-d)$ for NC-dye pairs, where the emission of the acceptor is recorded simultaneously with the

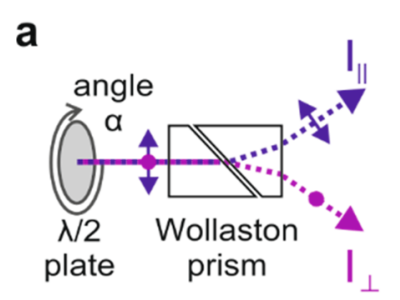

b
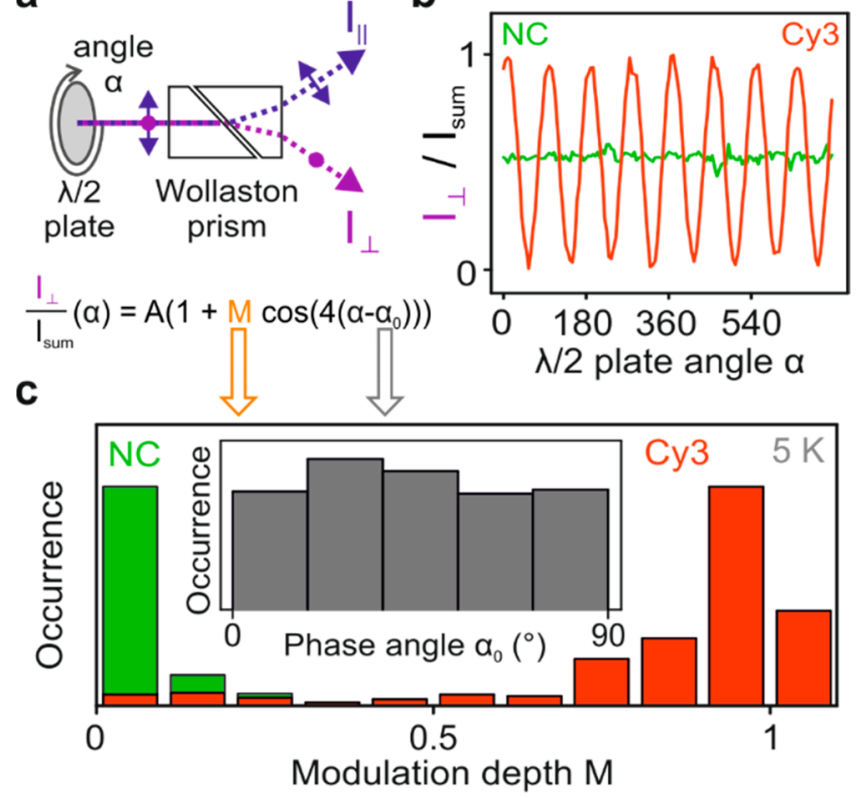

Figure 3. Polarization anisotropy of the PL of single NCs and $\mathrm{Cy} 3$ molecules excited by EET at $5 \mathrm{~K}$. (a) Schematic of the measurement methodology. A half-wave plate is rotated in the PL pathway before the PL is split into two orthogonally polarized channels $I_{\perp}$ and $I_{\| \cdot}$ (b) Examples of PL intensity modulation traces for a single NC (green) and a Cy3 dye molecule (red). The intensity of one polarization channel is divided by the sum of both channels $\frac{I_{\perp}}{I_{\text {sum }}}$ and plotted against the half-wave plate angle. Fitting the curves with Malus' law $\frac{I_{\perp}}{I_{\text {sum }}}(\alpha)=A \times\left(1+M \cos \left(4\left(\alpha-\alpha_{0}\right)\right)\right)$ yields the modulation depth $M$, where $M=0$ corresponds to unpolarized emission and $M=1$ results from linearly polarized emission. (c) Histogram over the modulation depths of different NCs (green) and EET-pumped Cy3 molecules (red). While NCs show unpolarized emission, Сy3 molecules display $M$ values close to unity. Values above unity arise from noise in the background correction. The inset shows the distribution of effective single-molecule phase angles, defined in panel (a), demonstrating that the single molecules are distributed uniformly in space.

residual luminescence of the donor. In panel (b), a situation observed in approximately $80 \%$ of all cases, the NC emission is stable over a time of almost $80 \mathrm{~s}$, exhibiting merely a brief jump at time $20 \mathrm{~s}$ (marked by a yellow asterisk). Interestingly, the brief jump in the NC spectrum appears to coincide with a jump in the dye spectrum. The dye emission, in contrast to the NC PL, shows broad spectral meandering over a range of 6 $\mathrm{nm}$. The zero-phonon lines of NC and dye PL are separated, on average, by $120 \mathrm{meV}$, but even such spectral jumps appear to have no discernible effect on the efficiency of EET and the brightness of the dye acceptor. The same is true for the situation in panel (c), where donor and acceptor resonances are now separated by $160 \mathrm{meV}$. At a time of $25 \mathrm{~s}$ (yellow asterisk) into the measurement the dye is quenched temporarily. EET is shut off, and the NC emission brightens. Such a distinct anticorrelation in emission intensities, analogous to the room-temperature result in Figure 1c, is observed in only $3 \%$ of all cases. Finally, the case in panel (d) shows spectral jumps of the NC by almost $30 \mathrm{meV}$ and subnanometer-width zero-phonon lines of the dye. Despite these large discrete jumps in spectral position of the donor, no correlated changes of the acceptor emission intensity are 

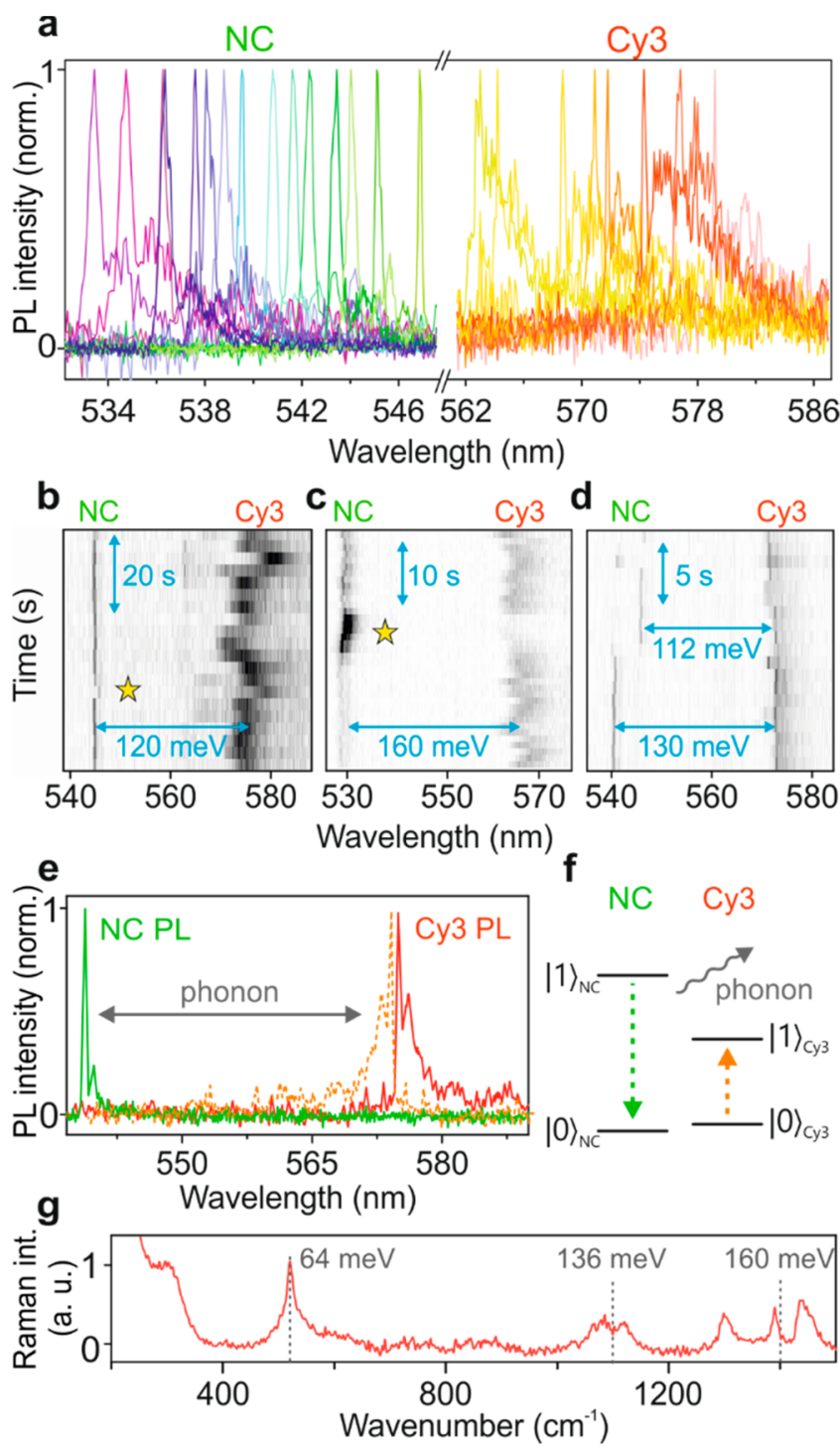

Figure 4. Spectra and spectral dynamics of single NCs and single EET-pumped $\mathrm{Cy} 3$ molecules at $5 \mathrm{~K}$. (a) Examples of normalized spectra of individual NCs (left) and Cy3 molecules (right). Spectra scatter over $12 \mathrm{~nm}$ for the NCs and $20 \mathrm{~nm}$ for the dye molecules, but generally show narrow line widths of below $1 \mathrm{~nm}$. $(\mathrm{b}-\mathrm{d})$ Examples of spectral evolution of the dual emission of individual NC/Cy3 pairs at $5 \mathrm{~K}$. The time axis originates at the intercept with the wavelength axis. Notable features are marked by an asterisk. (b) Cy3 emission can show spectral diffusion over a range of several nanometers while the NC peak position remains stable over time. (c) Fluctuations in EET efficiency can lead to anticorrelated intensities. (d) Even a large spectral jump of the NC does not affect $\mathrm{Cy} 3$ emission. (e) Example time-averaged spectra of a NC (green) and a Cy3 molecule (red), shifted to the average spectral positions of the NC and Cy3 dye PL peaks to remove the effect of spectral diffusion. The $\mathrm{Cy} 3$ absorption spectrum is approximated by the mirrored PL spectrum (dashed orange line). (f) Schematic of nonresonant energy transfer involving simultaneous emission of a phonon in the NC and a virtual photon driving dipole-dipole coupling. (g) Raman spectra of $\mathrm{FAPbBr}_{3} \mathrm{NCs}$ (exc. $780 \mathrm{~nm}$ ). Raman modes, predominantly due to the organic species, range up to $160 \mathrm{meV}$ in energy, implying that phonon emission can account for the nonresonant EET observed.

observed. We conclude that EET from the NC to the dye cannot be limited by the spectral overlap between donor and acceptor.
Figure $4 \mathrm{e}$ shows a closer inspection of example spectra of donor and acceptor. Again, the single NC residual donor PL is measured simultaneously with the acceptor PL. To estimate the acceptor absorption spectrum, to a first approximation, we can simply consider the mirror image of the acceptor PL spectrum, which is plotted as an orange dashed line. A small shift is expected between the zero-phonon peak in absorption and emission due to the structural relaxation of the dye molecule in the excited state. In room-temperature ensemble absorption and emission measurements, this shift amounts to $12 \mathrm{~nm}$, but this value will be much smaller in frozen solids where the molecules adopt equilibrium conformations. ${ }^{34}$ Clearly, there is no appreciable overlap between the donor emission and the acceptor absorption.

While the coupling of excitons to phonons in perovskite nanostructures is generally quite weak, as seen in the fact that there is no discernible vibronic progression in the NC PL-in contrast to the case of $\mathrm{CdSe} \mathrm{NCs}^{33}$ - the coupling of electrons to phonons is not. The most dramatic visualization of this effect comes from the identification of up to fourth-order multiphonon transitions in Raman scattering from purely inorganic perovskite $\mathrm{NCs}$, as well as the demonstration of polaronic features in photoelectron spectra. ${ }^{35}$ Figure $4 \mathrm{~g}$ shows Raman spectra of the NCs used here, excited at $780 \mathrm{~nm}$. The dominant optical phonon of these nanocrystals is observed at around $160 \mathrm{meV}$, which is higher than in purely inorganic perovskite NCs. ${ }^{33}$ Many lower-energy modes also exist, which can mix with the high-frequency vibration. As sketched in panel (f), we propose that phonon emission, promoted by the strong Fröhlich-type electron-phonon coupling in the polar lattice, ${ }^{36}$ permits efficient dissipation of excitation energy within the NC, thereby enabling nonresonant EET to the acceptor. Strong electron-phonon coupling ${ }^{36,37}$ has dramatic consequences on electron cooling and shows up in ultrafast two-dimensional spectroscopy. ${ }^{38}$ This rapid cooling is particularly interesting, since incoherent pump-probe experiments have been interpreted to show slow cooling, ${ }^{39}$ which could potentially be favorable to carrier extraction in photovoltaic applications. Our experiments are clearly incompatible with the notion of slow carrier cooling; nonresonant EET implies the simultaneous emission of a phonon and a virtual photon from the acceptor, ${ }^{9}$ necessitating strong coupling of carriers to phonons.

Finally, we note that, conceivably, either direct tunneling from the NC to the dye or a Dexter-type transfer could contribute to EET. However, Dexter transfer, which is known to occur between molecules in the triplet state and between closely spaced NCs is still, strictly, a resonant process, requiring spectral overlap. ${ }^{40}$ Although both donor NCs and acceptor molecules are polar and could conceivably undergo some binding, the dense NC ligands should prevent too close a proximity. Crucially, we see no difference in the PL spectrum and lifetime of single dye molecules excited either directly or indirectly by EET, ${ }^{25}$ implying that a charge-transfer tunnelingtype interaction between donor and acceptor is very unlikely. Ultimately, of course, it would be desirable to design model donor-acceptor complexes with variable NC-dye spacing, using, e.g., DNA spacers. ${ }^{27}$ It will be very challenging to decorate the large NC surface area with precisely one acceptor using conventional bioinspired functionalization techniques.

EET from single $\mathrm{FAPbBr}_{3}$ perovskite nanocrystals to single dye molecules does not occur by FRET, since-in contrast to pure dye aggregates or assemblies of CdSe-based NCs and dye 
molecules ${ }^{23}$ - there is no discernible effect of spectral line width and donor-acceptor spectral overlap. The only way that EET can become so efficient is by electron-phonon coupling, which enables nonresonant EET but which does not show up in a vibronic progression of the excitonic fluorescence. Nonresonant EET is highly efficient on the level of single donors and acceptors, suggesting a range of applications in exciton recycling ${ }^{41}$ and luminescent solar concentrators. ${ }^{42} \mathrm{~A}$ precise understanding of the nature of EET is also crucial in exploiting donor-acceptor systems as molecular rulers in biophysical applications. We stress here that only cryogenic investigations, which allow a direct control of the intrinsic line width and thus the spectral overlap, can quantitatively discern alternative EET mechanisms from the common FRET. Such studies should routinely be part of benchmarking the precision and accuracy of single-molecule FRET rulers. ${ }^{43}$

Experimental Methods. The synthesis of the NCs is described in ref 25. Ensemble spectra were taken in toluene solution. Samples with immobilized NC/dye pairs were prepared by spin coating a $\sim 100 \mathrm{~nm}$ thick film of PMMA from a solution of PMMA, NCs, and either Cy3 (Cyanin 3 NHS-ester from Lumiprobe, article number 11020) or Cy5 (Cyanin 5 NHS-ester from Lumiprobe, article number 13020) in toluene at nanomolar concentrations onto a transparent substrate (glass for room-temperature measurements, sapphire for low-temperature measurements). Fluorescence microscope images were taken with a confocal scanning microscope as described in detail elsewhere. ${ }^{25}$ Excitation light was provided by a $405 \mathrm{~nm}$ laser pulsed at $2.5 \mathrm{MHz}$. Time traces of individual $\mathrm{NC} / \mathrm{Cy} 5$ pairs were acquired by splitting the $\mathrm{PL}$ in a $\mathrm{NC}$ and a dye channel by using a $600 \mathrm{~nm}$ dichroic mirror and suitable bandpass filters. Both channels were then detected in separate Hanbury Brown and Twiss photon correlators consisting of a 50:50 beam splitter and two avalanche photodiodes (APDs) each. Spectra of individual spots were taken with a grating spectrometer and a CCD camera. All measurements were carried out under nitrogen atmosphere. Overlap integrals were calculated using the "ale" UV-vis-IR spectral software package.

For temperature-dependent measurements, the sample was mounted in a cold-finger helium-flow cryostat. A $400 \mathrm{~nm}$ pulsed frequency-doubled femtosecond laser was used for widefield excitation with a power of $600 \mathrm{~W} / \mathrm{cm}^{2}$. Background fluorescence and, depending on the experiment, residual NC emission was blocked by suitable filters. For determining the number of spots per unit area, PL was collected with an sCMOS camera, and spots were counted by an automated algorithm. Spectra of individual spots were resolved by a grating spectrometer and recorded with a back-illuminated CCD camera.

\section{AUTHOR INFORMATION}

\section{Corresponding Author}

*E-mail: john.lupton@ur.de.

\section{ORCID}

Dmitry N. Dirin: 0000-0002-5187-4555

Maksym V. Kovalenko: 0000-0002-6396-8938

John M. Lupton: 0000-0002-7899-7598

\section{Author Contributions}

The manuscript was written with contributions of all authors. All authors have given approval to the final version of the manuscript.

\section{Notes}

The authors declare no competing financial interest.

\section{ACKNOWLEDGMENTS}

This work was supported by the German Science Foundation through project B03 in the Sonderforschungsbereich 1277. In addition, we thank Dr. Rolf Erni for providing HRTEM images and Nadjafi Manouchehr for assistance with Raman measurements.

\section{REFERENCES}

(1) Metivier, R.; Nolde, F.; Müllen, K.; Basché, T. Electronic excitation energy transfer between two single molecules embedded in a polymer host. Phys. Rev. Lett. 2007, 98 (4), 047802.

(2) Fueckel, B.; Koehn, A.; Harding, M. E.; Diezemann, G.; Hinze, G.; Basché, T.; Gauss, J. Theoretical investigation of electronic excitation energy transfer in bichromophoric assemblies. J. Chem. Phys. 2008, 128 (7), 074505.

(3) List, E. J. W.; Creely, C.; Leising, G.; Schulte, N.; Schluter, A. D.; Scherf, U.; Müllen, K.; Graupner, W. Excitation energy migration in highly emissive semiconducting polymers. Chem. Phys. Lett. 2000, 325 (1-3), 132-138.

(4) Holstein, T.; Lyo, S. K.; Orbach, R. Phonon-assisted radiative transfer. Phys. Rev. B 1977, 16 (2), 934-942.

(5) Kowerko, D.; Schuster, J.; Amecke, N.; Abdel-Mottaleb, M.; Dobrawa, R.; Würthner, F.; von Borczyskowski, C. FRET and ligand related NON-FRET processes in single quantum dot-perylene bisimide assemblies. Phys. Chem. Chem. Phys. 2010, 12 (16), 41124123.

(6) Stupak, A. P.; Blaudeck, T.; Zenkevich, E. I.; Krause, S.; von Borczyskowski, C. The nature of non-FRET photoluminescence quenching in nanoassemblies from semiconductor quantum dots and dye molecules. Phys. Chem. Chem. Phys. 2018, 20 (27), 18579-18600.

(7) Vekshin, N. Energy Transfer in Macromolecules; SPIE Press Book: 1996; Vol. PM26.

(8) Vuojola, J.; Hyppanen, I.; Nummela, M.; Kankare, J.; Soukka, T. Distance and temperature dependency in nonoverlapping and conventional Förster resonance energy-transfer. J. Phys. Chem. B 2011, 115 (46), 13685-13694.

(9) Van Uitert, L. G.; Johnson, L. F. Energy transfer between rareearth ions. J. Chem. Phys. 1966, 44 (9), 3514-3519.

(10) Vuojola, J.; Lamminmaki, U.; Soukka, T. Resonance energy transfer from lanthanide chelates to overlapping and nonoverlapping fluorescent protein acceptors. Anal. Chem. 2009, 81 (12), 5033-5038.

(11) Miller, M. P.; Wright, J. C. Multiphonon and energy transfer relaxation in charge compensated crystals. J. Chem. Phys. 1979, 71 (1), 324-338.

(12) Iaru, C. M.; Geuchies, J. J.; Koenraad, P. M.; Vanmaekelbergh, D.; Silov, A. Y. Strong carrier-phonon coupling in lead halide perovskite nanocrystals. ACS Nano 2017, 11 (11), 11024-11030.

(13) Steele, J. A.; Puech, P.; Keshavarz, M.; Yang, R.; Banerjee, S.; Debroye, E.; Kim, C. W.; Yuan, H.; Heo, N. H.; Vanacken, J.; Walsh, A.; Hofkens, J.; Roeffaers, M. B. J. Giant electron-phonon coupling and deep conduction band resonance in metal halide double perovskite. ACS Nano 2018, 12 (8), 8081-8090.

(14) Clapp, A. R.; Medintz, I. L.; Mauro, J. M.; Fisher, B. R.; Bawendi, M. G.; Mattoussi, H. Fluorescence resonance energy transfer between quantum dot donors and dye-labeled protein acceptors. J. Am. Chem. Soc. 2004, 126 (1), 301-310.

(15) Stöferle, T.; Scherf, U.; Mahrt, R. F. Energy transfer in hybrid organic/inorganic nanocomposites. Nano Lett. 2009, 9 (1), 453-456.

(16) Walker, B. J.; Nair, G. P.; Marshall, L. F.; Bulovic, V.; Bawendi, M. G. Narrow-band absorption-enhanced quantum dot/J-aggregate conjugates. J. Am. Chem. Soc. 2009, 131 (28), 9624-9625.

(17) Chen, Z. Y.; Berciaud, S.; Nuckolls, C.; Heinz, T. F.; Brus, L. E. Energy transfer from individual semiconductor nanocrystals to graphene. ACS Nano 2010, 4 (5), 2964-2968. 
(18) Funston, A. M.; Jasieniak, J. J.; Mulvaney, P. Complete quenching of CdSe nanocrystal photoluminescence by single dye molecules. Adv. Mater. 2008, 20 (22), 4274-4280.

(19) Harris, R. D.; Bettis Homan, S.; Kodaimati, M.; He, C.; Nepomnyashchii, A. B.; Swenson, N. K.; Lian, S. C.; Calzada, R.; Weiss, E. A. Electronic Processes within Quantum Dot-Molecule Complexes. Chem. Rev. 2016, 116 (21), 12865-12919.

(20) Hua, Z.; Xu, Q. F.; Huang, X. N.; Zhang, C. F.; Wang, X. Y.; Xiao, M. Energy transfer from a single semiconductor nanocrystal to dye molecules. ACS Nano 2014, 8 (7), 7060-7066.

(21) Muthu, C.; Vijayan, A.; Nair, V. C. $\mathrm{CH}_{3} \mathrm{NH}_{3} \mathrm{PbBr}_{3}$ perovskite nanocrystals as efficient light-harvesting antenna for fluorescence resonance energy transfer. Chem. - Asian J. 2017, 12 (9), 988-995.

(22) Zhou, D. J.; Piper, J. D.; Abell, C.; Klenerman, D.; Kang, D. J.; Ying, L. M. Fluorescence resonance energy transfer between a quantum dot donor and a dye acceptor attached to DNA. Chem. Commun. 2005, 38, 4807-4809.

(23) Becker, K.; Lupton, J. M.; Müller, J.; Rogach, A. L.; Talapin, D. V.; Weller, H.; Feldmann, J. Electrical control of Förster energy transfer. Nat. Mater. 2006, 5 (10), 777-781.

(24) Soujon, D.; Becker, K.; Rogach, A. L.; Feldmann, J.; Weller, H.; Talapin, D. V.; Lupton, J. M. Time-resolved Förster energy transfer from individual semiconductor nanoantennae to single dye molecules. J. Phys. Chem. C 2007, 111 (31), 11511-11515.

(25) Hofmann, F. J.; Bodnarchuk, M. I.; Protesescu, L.; Kovalenko, M. V.; Lupton, J. M.; Vogelsang, J. Exciton gating and triplet deshelving in single dye molecules excited by perovskite nanocrystal FRET antennae. J. Phys. Chem. Lett. 2019, 10 (5), 1055-1062.

(26) Yu, Y. J.; Kim, K. S.; Nam, J.; Kwon, S. R.; Byun, H.; Lee, K.; Ryou, J. H.; Dupuis, R. D.; Kim, J.; Ahn, G.; Ryu, S.; Ryu, M. Y.; Kim, J. S. Temperature-dependent resonance energy transfer from semiconductor quantum wells to graphene. Nano Lett. 2015, 15 (2), 896-902.

(27) Becker, K.; Rogach, A. L.; Feldmann, J.; Talapin, D. V.; Lupton, J. M. Energetic disorder limits energy transfer in semiconductor nanocrystal-DNA-dye conjugates. Appl. Phys. Lett. 2009, 95 (14), 143101.

(28) Park, Y. S.; Guo, S. J.; Makarov, N. S.; Klimov, V. I. Room temperature single-photon emission and lasing for all-inorganic colloidal perovskite quantum dots. ACS Nano 2015, 9 (10), 10386-10393.

(29) Müller, J. G.; Lemmer, U.; Raschke, G.; Anni, M.; Scherf, U.; Lupton, J. M.; Feldmann, J. Linewidth-limited energy transfer in single conjugated polymer molecules. Phys. Rev. Lett. 2003, 91 (26), 267403.

(30) Rainò, G.; Landuyt, A.; Krieg, F.; Bernasconi, C.; Ochsenbein, S. T.; Dirin, D. N.; Bodnarchuk, M. I.; Kovalenko, M. V. Underestimated effect of a polymer matrix on the light emission of single CsPbBr3 nanocrystals. Nano Lett. 2019, 19 (6), 3648-3653.

(31) Walter, M. J.; Borys, N. J.; van Schooten, K. J.; Lupton, J. M. Light-harvesting action spectroscopy of single conjugated polymer nanowires. Nano Lett. 2008, 8, 3330-3335.

(32) Utzat, H.; Shulenberger, K. E.; Achorn, O. B.; Nasilowski, M.; Sinclair, T. S.; Bawendi, M. G. Probing linewidths and biexciton quantum yields of single lead halide perovskite nanocrystals in solution. Nano Lett. 2017, 17 (11), 6838-6846.

(33) Neuhauser, R. G.; Shimizu, K. T.; Woo, W. K.; Empedocles, S. A.; Bawendi, M. G. Correlation between fluorescence intermittency and spectral diffusion in single semiconductor quantum dots. Phys. Rev. Lett. 2000, 85 (15), 3301-3304.

(34) Kiraz, A.; Ehrl, M.; Bräuchle, C.; Zumbusch, A. Low temperature single molecule spectroscopy using vibronic excitation and dispersed fluorescence detection. J. Chem. Phys. 2003, 118, 10821-10824.

(35) Puppin, M.; Polishchuk, S.; Colonna, N.; Crepaldi, A.; Dirin, D. N.; Nazarenko, O.; De Gennaro, R.; Gatti, G.; Roth, S.; Barillot, T.; Poletto, L.; Xian, R. P.; Rettig, L.; Wolf, M.; Ernstorfer, R.; Kovalenko, M. V.; Marzari, N.; Grioni, M.; Chergui, M. Evidence of large polarons in photoemission band mapping of the perovskite semi- conductor $\mathrm{CsPbBr}_{3}$. 2019, arxiv:1909.00248. arXiv.org e-Print archive. https://arxiv.org/abs/1909.00248 (accessed 2019).

(36) Bozyigit, D.; Yazdani, N.; Yarema, M.; Yarema, O.; Lin, W. M. M.; Volk, S.; Vuttivorakulchai, K.; Luisier, M.; Juranyi, F.; Wood, V. Soft surfaces of nanomaterials enable strong phonon interactions. Nature 2016, 531 (7596), 618-622.

(37) Schlipf, M.; Ponce, S.; Giustino, F. Carrier lifetimes and polaronic mass enhancement in the hybrid halide perovskite $\mathrm{CH}_{3} \mathrm{NH}_{3} \mathrm{PbI}_{3}$ from multiphonon Fröhlich coupling. Phys. Rev. Lett. 2018, 121 (8), 086402.

(38) Richter, J. M.; Branchi, F.; Valduga de Almeida Camargo, F.; Zhao, B.; Friend, R. H.; Cerullo, G.; Deschler, F. Ultrafast carrier thermalization in lead iodide perovskite probed with two-dimensional electronic spectroscopy. Nat. Commun. 2017, 8, 376.

(39) Li, M. J.; Bhaumik, S.; Goh, T. W.; Kumar, M. S.; Yantara, N.; Gratzel, M.; Mhaisalkar, S.; Mathews, N.; Sum, T. C. Slow cooling and highly efficient extraction of hot carriers in colloidal perovskite nanocrystals. Nat. Commun. 2017, 8, 14350.

(40) Hoffman, J. B.; Choi, H.; Kamat, P. V. Size-dependent energy transfer pathways in CdSe quantum dot-squaraine light-harvesting assemblies. Förster versus Dexter. J. Phys. Chem. C 2014, 118, 1845318461.

(41) Franzl, T.; Klar, T. A.; Schietinger, S.; Rogach, A. L.; Feldmann, J. Exciton recycling in graded gap nanocrystal structures. Nano Lett. 2004, 4 (9), 1599-1603.

(42) van Sark, W.; Barnham, K. W. J.; Slooff, L. H.; Chatten, A. J.; Buchtemann, A.; Meyer, A.; McCormack, S. J.; Koole, R.; Farrell, D. J.; Bose, R.; Bende, E. E.; Burgers, A. R.; Budel, T.; Quilitz, J.; Kennedy, M.; Meyer, T.; Donega, C. D. M.; Meijerink, A.; Vanmaekelbergh, D. Luminescent solar concentrators - a review of recent results. Opt. Express 2008, 16 (26), 21773-21792.

(43) Hellenkamp, B.; et al. Precision and accuracy of single-molecule FRET measurements-a multi-laboratory benchmark study. Nat. Methods 2018, 15, 669-678. 